Ljubljana

\title{
RENDEMENT STYLISTIQUE DE L'ELLIPSE DU PRONOM SUJET DANS LE "THÉSÉE" D’ANDRÉ GIDE
}

Répétition et non-répétition du pronom sujet dans la coordination en français moderne. Coordinations copulatives dans le Thésée de Gide. Juxtaposition. Ellipse du sujet et de l'auxiliaire. Propositions affirmatives et négatives coordonnées. Propositions à des temps et modes différents.

Thésée, le dernier récit de Gide, projeté depuis 1931, ne fut achevé qu'en 1944. Il n'est pas vaste, une quarantaine de pages dans l'édition de la Pléiadle. Mais la verve avec laquelle Gide, à l’âge de 75 ans, a su traiter et renouveler le vieux mythe est surprenante, surprenante aussi son habileté à utiliser des moyens stylistiques très variés pour accentuer des tons très divers qu' exigeait son sujet. Un de ces moyens stylistiques est l'ellipse du sujet dans des propositions coordonnées, quand ce sujet est commun à deux ou plusieurs verbes.

$D$ 'après les règles de bon usage du français moderne, le sujet est répété au moyen du pronom personnel quand il $\mathrm{y}$ a, dans une phrase, plusieurs verbes qui ont un sujet commun et qui sont juxtaposés ou coordonnés par des conjonctions et, ou, ni, mais, etc.; mais le sujet peut être exprimé une seule fois quand les propositions sont du même type. La répétition est donc obligatoire avec deux présentations différentes de l'idée: quand les verbes de la coordination sont à des temps ou modes différents, quand une proposition affirmative juxtaposée suit une proposition négative: J'allais sortir et je me suis apercu ... ; Je vais $\mathrm{m}^{\prime}$ en aller, mais je voudrais d'abord ... ; Je ne sors pas, je rentre. La répétition est

1 Les exemples cités sont pris dans l'ouvrage d'Albert Dauzat, Grammaire raisonnée de la langue française, Lyon, IAC, 1947. p. 265 . 
obligatoire aussi quand il y a l'inversion du sujet. Elle n'est que facultative quand les verbes sont aux mêmes temps on modes et quand une proposition négative suit une proposition affirmative: $\underline{\text { Il ouvrit la }}$ porte et il sortit ou Il ouvrit la porte et sortit; II était en colère, mais il ne répondait pas ou Il était en colère, mais ne répondait pas; Elle écoute, elle entend, elle espère ou Elle écoute, entend, espère ${ }^{2}$. Nous ajouterons que, dans les trois exemples cités qui concernent le sujet non-répété, la distance entre les verbes est brève, ils sont séparés tout au plus par un complément.

Dans les cas ou les deux usages sont permis - la répétition et l'omission du sujet - le choix de l'un ou de l'autre confère à la phrase une nuance stylistique particulière. Quand on répète le sujet, l'on insiste sur la personne qui fait les actions; quand on omet la répétition, l'accent est sur les verbes et ce qui est mis en relief, c'est le lien étroit entre les actions; le second verbe exprime alors une action qui complète la première ou en est la suite naturelle, ou bien s'oppose à elle ${ }^{3}$. Ferdinand Brunot dit excellemment: "Dans notre langue actuelle, il faut, pour ne point donner un sujet à un verbe, qu'il soit intimement lié à un autre, de telle façon que les diverses actions soient pour ainsi dire les portions, les phases $d^{\prime}$ une action d'ensemble." 4

Dans une première série d'exemples tirés du Thésée de Gide, deux propositions sont coordonnées au moyen des conjonctions ou adverbes et,

2 Exemples cités par A. Dauzat, op. cit., p. 266.

3 Cf. Grammaire Larousse du $\mathrm{Xx}^{\mathrm{e}}$ siecle, Paris, Larousse, 1936, pp. 181-182.

4 Ferdinand Brunot, La pensée et la langue, Paris, Masson et Cie, 1953 , p. 279. 
et même, puis, ou. Les deux verbes sont placés parfois assez près l'un de l'autre, dans d'autres cas ils sont séparés par plusieurs compléments ou propositions. Le temps et le mode sont les mêmes dans les deux propositions, leur forme est affirmative. Le rapprochement des deux verbes par l'ellipse du pronom sujet met en relief le lien logique ou affectif entre eux: le second renforce ou explique la signification de l'action qu'exprime le premier ou indique une action qui en est la conséquence:

Minos sourit derechef et donna des ordres, en sorte que les gardes n' emmenassent que mes compagnons. (1421)

Ici, le premier verbe n'est suivi que d'un adverbe, la distance entre les deux verbes coordonnés est donc brève, l'ellipse du pronom sujet devant le second verbe le rapproche davantage du premier et montre qu'il est lié à lui par son sens: Minos, qui avait aperçu l'intérêt de sa fille pour Thésée, sourit avec bienveillance et par conséquent donna l'ordre de ne pas emmener Thésée.

Voilà un exemple semblable où le premier verbe de la coordination n'est suivi que d'un complément d'objet, les deux verbes rapprochés au moyen de l'ellipse du sujet sont apparentés par leur sens ${ }^{6}$ :

Mais, dès qu'A riane paraissait, je détournais les yeux et dissimulais de mon mieux ... (1440)

5 Les chiffres accompagnant chaque citation de Thésée correspondent aux pages du volume: André Gide, Romans, Récits et Soties. Oeuvres lyriques, Paris, Gallimard, "Bibliothèque de la Pléiade", 1969.

6 Pour saisir le sens complet des verbes de la coordination, il faut prendre en considération - dans le cas présent et ailleurs non seulement ces verbes, mais aussi les compléments qui les accompagnent. 
Dans le cas qui suit, la distance entre les deux verbes est un peu plus prononcée:

Des brigands de tout poil recommençaient d'infester le pays et $s$ 'en donnaient à joie ... (1418-9)

Le premier verbe est suivi d'un infinitif et de son complément d'objet direct. Le second verbe, devant lequel le sujet n'est pas répété, en complète le sens, indiquant l'atmosphère dans laquelle l'action s'accomplit.

Il y a une pareille distance entre les verbes dans la phrase où Thésée dit à Oedipe:

Je reste enfant de cette terre et crois que l'homme ... doit faire jeu des cartes qu'il a. (1453)

Dans ce cas-là le premier verbe est suivi d'un attribut accompagné d'un complément déterminatif. La distance entre les verbes n'est pas excessive et la sensation qu'ils sont unis par leur sens reste complète.

Dans le cas qui suit, la distance est produite par un groupe adverbial qui précède le second verbe et fait l'effet d'une coupe. Il s'agit d'Oedipe qui parle à Thésée:

Il se tut et, durant quelques instants, resta plongé

dans une méditation profonde. (1451)

Malgré cette coupe, le second verbe reste lié par son sens au premier, il accompagne son action, il en indique la fin immédiate: Oedipe se tut pour pouvoir méditer.

Dans d'autres cas la distance entre les deux verbes est considérable, p. ex. :

Pourtant Pirithoüs se montra bientôt fort confus de cette dépravation passagère et promit de se racheter à ses propres yeux et aux miens par un excès de zèle. (1440) 
Le premier verbe est suivi de trois groupes grammaticaux qui le séparent du second: un adverbe de temps (bientôt), un attribut du sujet (fort confus) et un complément de cet attribut (de cette dépravation passagère). Mais le second verbe est suivi, lui aussi, d'une série de compléments, ce qui garantit à la phrase un équilibre harmonieux. Un tel équilibre $n$ 'a pas seulement une valeur acoustique, mais aussi une valeur logique et affective. Il permet aux deux verbes, dont le second exprime une conséquence de $\mathrm{I}^{\prime}$ action du premier, de rester liés malgré la distance.

Le lien logique entre les verbes, quand il est renforcé par un tel parallélisme, est encore mieux visible dans le passage où Pasiphaë parle de son mari Minos:

Il soutient qu'il faut d'abord avoir compris pour bien juger et pense qu'il ne sera bon juge qu'après qu'il aura tout éprouvé ... (1427)

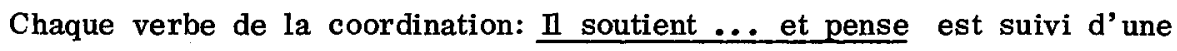
proposition substantive (qu'il faut d'abord avoir compris .... qu'il ne sera bon juge que) qui englobe une proposition subordonnée du second degré, la première une proposition infinitive à valeur finale (pour bien juger), la seconde une proposition temporelle (après qu'il aura tout éprouvé). Il y a donc un parallélisme presque complet dans la construction des deux parties de la phrase. Les deux verbes des propositions principales ont un sens très proche et les deux propositions substantives expriment la même idée, la première d'une manière générale et impersonnelle, la seconde comme une conclusion appliquée à Minos.

Une construction parallèle, on la rencontre aussi dans les deux propositions coordonnées d'une phrase qui décrit la scène entre Thésée et Ariane à l'entrée du labyrinthe. Il s'agit des pelotons que Dédale a remis à Thésée. C'est à propos de ces pelotons qu'entre Ariane et Thésée s'élève leur première dispute. Et Thésée explique: 
Elle voulut que je lui remette, et prétendit garder en son giron lesdits pelotons .... (1438)

Les deux verbes voulut ... et prétendit au sens très proche sont suivis chacun d'une proposition - complément d'objet dont la deuxième est une proposition infinitive. Les verbes des deux propositions subordonnées ont le même objet (les pelotons) qui pourtant n'est pas exprimé dans la première. Il se produit ainsi, avant la conjonction qui lie les deux parties de la coordination, une espèce d'hiatus, une attente qui n'est satisfaite qu'à la fin de la seconde proposition qui amène finalement l'objet en question - les pelotons, cause de la discorde.

La distance entre les verbes de la coordination est remarquable dans une phrase au début du récit; Thésée y explique comment Hippolyte, tout en étant fils du roi, s'en souciait peu:

Mais Hippolyte $s^{\prime}$ en souciait peu; moins encore que je ne faisais à son âge et, comme moi dans ce temps, se passait fort commodément de le savoir. (1415)

Les deux propositions principales: Hippolyte $\cdot s^{\prime}$ en souciait peu ... et ... se passait fort commodément de le savoir, sont séparées par une proposition subordonnée comparative qui dépend de la première principale (moins encore que je ne faisais à son âge) et une subordonnée où le verbe est omis, qui marque aussi un rapport de comparaison et qui dépend de la seconde proposition principale (comme moi dans son temps). Pour rendre cette distance plus visible, Gide a recours au point-virgule après la première principale. L'ellipse du sujet à une telle distance, après deux propositions subordonnées qui tendent à établir un rapport de comparaison entre deux sujets différents: je (Thésée) et il (Hippolyte), cet il qui précisément n'est pas répété, invite le lecteur à faire un effort s'il veut saisir le fil du récit, et à revenir au début de la phrase pour retrouver le sujet. Si dans une construction où la succéssion des verbes est immédiate, cette proximité elle-même rappelle le lien logique ou affectif entre 
eux, dans le cas présent, où la distance est considérable, cette distance sollicite le lecteur de chercher ce lien.

La conjonction et peut être renforcée par même. Thésée parle de la légende qui s'est formée autour d'Ariane abandonnée à Naxos:

Je me gardais de démentir ces bruits d'ou je tirais un surcrôit de prestige; et même renchérissais sur les racontars afin d'ancrer le peuple en des croyances ... (1445)

La conjonction composée et même indique que l'action exprimée dans la première proposition (je me gardais de démentir) devient plus intense dans la seconde (renchérissais sur les racontars). La distance entre les deux verbes produite par l'intercalation d'une proposition infinitive de laquelle dépend une subordonnée locative est accentuée par l'emploi du point-virgule entre les deux groupes de propositions. Mais l'harmonie y est rétablie par un complément circonstanciel et une proposition infinitive à valeur finale qui complètent la seconde proposition coordonnée. La coupe entre les deux parties de la phrase et la construction équilibrée de ces deux parties servent à accentuer la gradation de l'action.

Une impression semblable se dégage d'une coordination dont la seconde proposition est introduite par l'adverbe puis qui met en relief la distance temporelle entre les deux actions dont la première prépare la seconde:

A mon retour en Crète, je m'entretins avec Minos de mes études et de mes voyages; puis lui fis part d'un projet que $j^{\prime}$ avais nourri ... (1432)

Les coordinations traitées jusqu'ici sont copulatives. Máis voilà deux exemples intéressants où deux propositions subordonnées sont coordonnées entre elles au moyen de la conjonction disjonctive ou après laquelle le sujet $n$ 'est pas répété:

Mais où que j'allasse ou demeurasse, je restais Grec. (1432) 
... je compris que la constance d'une amitié nous retient ou nous tire arrière. (1448)

Dans les deux cas les verbes sont tres rapprochés, forment corps et indiquent deux altérnatives, deux possibilités dont l'une exclut l'autre. Dans le premier cas il s'agit de deux possibilités opposées, dans le deuxième de deux possibilités qui représentent deux degrés d'intensité de la même action.

A côté des propositions coordonnées qui sont liées au moyen de conjonctions, nous trouvons dans le récit

sitions et, quand il $\mathrm{y}$ a plus de deux propositions coordonnées, des combinaisons des propositions juxtaposées avec celles liées à l'aide de conjonctions. La juxtaposition où le sujet n'est pas répété donne l'impression que les actions se succèdent avec un rythme serré.

Voilà deux propositions principales juxtaposées dans une phrase où Thésée parle des vapeurs qui $s^{\prime}$ exhalent dans le labyrinthe:

Elles procurent une ivresse pleine de charme et prodigue de flatteuses erreurs, invitent à certaine activité vaine le cerveau ... (1433)

Après le très long et compliqué complément d'objet (une ivresse pleine de charme et prodigue de flatteuses humeurs) qui sépare les deux verbes, il y a une pause qui invite le lecteur à revenir à la signification de l'action qui précède et qui est reprise et précisée dans la seconde proposition de la coordination.

Dans un autre passage trois propositions coordonnées, dont deux juxtaposées et la troisième introduite par et, expriment trois actions qui se suivent à brève distance: 
... je ceignis autour de mes reins cette écharpe, la

passai entre mes cuisses et, la ramenant par devant,

l'assujettis. (1424)

Dans l'exemple qui suit, il y a trois propositions juxtaposées et une quatrième introduite par l'adverbe puis:

Au débarqué, des gardes armés nous entourèrent, s'emparèrent de mon glaive et de celui de Pirithoüs, s'assurèrent que nous ne portions point sur nous $d$ 'autres armes, puis nous emmenèrent pour comparaître devant le roi ... (1420)

Les trois actions exprimées par les premiers trois verbes se succèdent rapidement, ce qui est souligné soit par l'emploi du passé simple soit par la non-répétition du sujet soit par l'absence de la conjonction; la dernière proposition introduite par l'adverbe puis qui indique une brève distance temporelle exprime une action en vue de laquelle toutes les précédentes ont été accomplies.

Dans les coordinations examinées les verbes sont dans un temps simple. Mais dans le récit sont fréquents aussi des exemples où les verbes de la coordination sont dans un temps composé. Gide pratique alors, avec l'ellipse du sujet, l'ellipse de l'auxiliaire. Elle est possible, d'après le bon usage actuel, quand les verbes sont au même temps, au même mode et à la même forme (affirmative ou négative) ou quand le deuxième est dans la forme négative construite au moyen de non ${ }^{7}$.

Voilà l'ellipse du pronom sujet et de l'auxiliaire dans une juxtaposition où Thésée parle de son ami Pirithoüs:

Il m'avait, au temps de ma jeunesse, accompagné partout, beaucoup aidé. (1448)

7 Cf. Maurice Grevisse, Le Bon usage, Gembloux-Paris, DuculotGeuthner, $7^{e}$ éd., 1961, p. 578. 
Les deux verbes au plus-que-parfait sont proches par leur sens, leurs participes passés sont séparés par deux adverbes - partout, beaucoup qui produisent une impression acoustique semblable, ce qui contribue à souligner le lien entre eux. Le pronom sujet et l'auxiliaire qui appartiennent à l'un et à l'autre participe sont placés au début de la phrase et séparés des participes par un complément circonstanciel assez long (au temps de ma jeunesse) qui se rapporte aux deux verbes. Tout cela sert à accentuer la parenté des deux actions et leur coïncidence dans le temps.

Dans une autre juxtaposition où les verbes sont encore au plus-queparfait, le premier verbe se trouve répété comme infinitif - complément du second verbe coordonné. Par ce jeu de mots l'action, exprimée dans la première proposition, est rectifiée, voire intensifiée dans la seconde:

Jusqu'alors j'avais tout incliné, vu tous s'incliner devant moi. (1450)

Dans une coordination de trois propositions dont les verbes sont au plusque-parfait, deux sont juxtaposées et la troisième liée au moyen de la conjonction et:

Il avait tenu tête au Sphinx; dressé l'Homme en face de l'énigme et osé l'opposer aux dieux. (145l)

Il s'agit d'Oedipe et les trois verbes indiquent trois de ses actions méritoires qui sont de la même valeur.

Nous trouvons une combinaison des propositions coordonnées où le sujet est répété et de celles où il ne l'est pas dans la longue phrase qui suit:

... je crois avoir rendu quelques notoires services; j'ai définitivement purgé la terre de maints tyrans, bandits et monstres; balayé certaines pistes aventureuses .... ; clarifié le ciel de manière que 1'homme, au front moins courbé, appréhendât moins la surprise. (1417) 
La première proposition dont le verbe est au présent est détachée des trois autres dont les verbes sont au passé composé. Cette coupure est produite par la répétition du pronom sujet devant le deuxième verbe, tandis que dans la troisième et la quatrième proposition il y a l'ellipse du sujet et de l'auxiliaire, ce qui les lie étroitement à la deuxième proposition. L'effet produit par cette construction est bien clair: dans la première proposition Thésée affirme ses mérites, les trois qui suivent servent à appuyer cette affirmation.

Très intéressante est une phrase où deux propositions temporelles juxtaposées dépendent de trois propositions principales, elles aussi juxtaposées. Dans les trois propositions principales le verbe est au passé simple, le sujet n'est pas répété, dans les deux subordonnées le verbe est au passé antérieur, le sujet et l'auxiliaire ne sont pas répétés:

Quand elle eut éventé ma ruse, découvert sa soeur sous le revêtement de Glaucos, elle mena grand raffut, poussa force cris rythmés, me traita de perfide ... (1444)

Dans chacune des deux coordinations l'ellipse rapproche les actions des verbes voisins par leur sens: eut éventé - découvert; mena - me traita poussa.

Toutes les coordinations traitées dans les paragraphes précédents sont affirmatives. Mais les exemples où les propositions à la forme affirmative et celles à la forme négative voisinent dans la même coordination, sans que le sujet soit répété, sont fréquents.

Dans les cas où une proposition affirmative est suivie d'une négative dans laquelle il y a l'ellipse du sujet, la proposition négative complète le verbe de la proposition affirmative parce qu'elle nie l'existence d'un fait qui serait opposé à cette affirmation. Thésée parle de Pirithoüs: 
Il $\mathbf{m}$ 'engageait à me soucier de Phèdre davantage et, sur ce point du moins, n'avait pas tort. (1448)

Ici aussi, comme Gide aime le faire, les deux propositions sont séparées par plusieurs compléments.

Dans une autre coordination avec et -

... je m'en fus visiter des pays lointains, me mettre à

l'école de savants étrangers, et ne les quittai point

qu'ils eussent encore à m'apprendre. (1432)

- le verbe de la premiere proposition (je m'en fus) est complété par deux propositions infinitives juxtaposées. La seconde proposition de la coordination qui est négative (ne les quittai point) ne complète la première que par rapport à sa deuxième proposition infinitive (me mettre à l'école des savants étrangers).

Voilà une construction assez compliquée où nous rencontrons la conjonction adversative mais:

Puis, joignant l'exemple aux paroles, je me démis aussitôt de toute autorité royale, rentrai dans le rang, ne craignis pas de me montrer sans escorte aux yeux de tous et comme un simple citoyen; mais $m$ 'occupai sans relâche de la chose publique, assurant la concorde, veillant à l'ordre de l'État. (1446)

Les verbes des trois propositions juxtaposées dont la troisième est négative expriment trois actions successives, presque contemporaines. Le sens de ces verbes est assez proche, car le troisième nie 1'existence d'une action qui pourrait anéantir les deux précédentes. La quatrième proposition introduite par mais indique une action qui d'habitude ne va pas de pair avec les trois précédentes.

Voilà encore une phrase de Thésée où il décrit l'aspect physique de Dédale:

Il est de très haute stature, non courbé malgré son grand âge; porte une barbe plus longue encore que celle de Minos ... (1430) 
Le sujet et le verbe copule de la première proposition (il est de très haute stature) ne peuvent pas être répétés devant la négation non dans non courbé malgré son âge. Deux particularités qui concernent la stature de Dédale sont ainsi énumérées dans une succession tres étroite. L'indication de la troisième particularité physique de Dédale, sa longue barbe, se trouve un peu détachée des deux précédentes: il y a bien dans la troisième proposition juxtaposée 1'ellipse du sujet, tandis que le verbe est exprimé.

Dans les coordinations où la premiere proposition est négative, la seconde, qui est affirmative indique une conséquence logique provenant de la négation de la première action. Phèdre dit à Thésée:

Je n'ignore pas ce qui vous amène ici et tiens à prévenir une erreur. (1426)

Plus compliquée est la phrase suivante prononcée par Thésée:

Mais, bien que Grec, je ne me sens aucunement porté vers ceux de mon sexe, si jeunes et charmants qu'ils puissent être, et diffère en cela d'Hercule ... (1441)

Dans cette phrase les deux verbes (je ne me sens aucunement - diffère) sont séparés l'un de l'autre par un long attribut (porté vers ceux de mon sexe) et par une assez vaste proposition concessive (si jeunes et charmants qu'ils puissent être). Après la coupure produite par cette intercalation, le verbe diffère met en relief la particularité de Thésée, indiquée en forme négative déja dans la première proposition.

Dans une autre longue phrase l'équilibre est établi entre les deux parties. Thésée parle de sa conviction que les cendres d'Oedipe porteront la bénédiction au sol où il reposera:

Je $n$ 'ajoutai point que ce qui $m$ 'importait c'est que ce sol fut celui de l'Attique, et me félicitai que les dieux aient su faire aboutir Thèbes à moi. (1453) 
Chacune des deux propositions coordonnées, l'une négative, l'autre positive (Je n'ajoutai point - et me félicitai) est suivie d'une subordonnée substantive qui est, à son tour, complétée par une proposition substantive du second degré - dans la dernière subordination c'est une proposition infinitive. Le rapport entre les verbes des propositions principales exprime moins la conséquence que l'opposition, on remplacerait aisément la conjonction et par mais.

Plusieurs fois on rencontre dans le récit de Gide la locution restrictive ne...que, soit dans la première soit dans la seconde proposition de la coordination où le sujet n'est pas répété. Elle porte sur un complément de la respective proposition.

Je plongeai, dûment entraîné, profondément, et ne reparus à la surface qu'après avoir sorti de l'escarcelle une agate onyx et deux chrysoprases. (1424)

Je sais bien que tout passe; mais ne m'occupe que du présent. (1429)

Dans les deux exemples cités, les conjonctions de la coordination sont une fois et, l'autre fois mais. La locution de restriction porte dans les deux cas sur un complément de la seconde proposition, dans notre premier cas sur une proposition infinitive temporelle, dans le second sur un complément d'objet. Dans les deux cas, il y a entre les verbes coordonnés une distance prononcée, produite par plusieurs compléments du verbe de la première proposition.

Dans une autre coordination avec mais, la locution restrictive se trouve dans la première proposition. Thésée parle du peu de poids que ses amours ont eu dans sa vie:

Celles-ci n'ont du reste eu d'importance que dans la première partie de ma vie; mais $m$ 'ont appris du moins à me connaître ... (14l5) 
L'ellipse du sujet après la conjonction adversative renforce l'impression que malgré la restriction exprimée dans la première proposition quelque chose subsiste.

Dans les exemples examinés jusqu'ici les verbes des propositions coordonnées sont à des temps et modes identiques, ce qui est, selon les règles du français contemporain, une condition fondamentale de la nonrépétition du pronom sujet. Mais Gide omet parfois le pronom sujet dans la deuxième proposition quand le temps de son verbe est différent de celui qui précède:

Nous abordâmes, un matin de mars, à Amnisos, petite bourgade qui sert de port à la proche Cnossos, ville capitale de l'île où Minos réside et a fait édifier son palais. (1420)

C'est la dernière partie de la phrase qui nous intéresse: $\ldots$ où Minos réside et a fait édifier son palais. Il s'agit de deux propositions locatives mais coordonnées entre elles. Les deux verbes sont à deux temps différents, le présent et le passé composé Ces deux temps ont pourtant entre eux un lien, le passé composé indique une action passée dont l'effet se prolonge au présent. Contrairement à la plupart des coordinations citées dans les paragraphes précédents où l'auteur a eu la tendance à intercaler entre les verbes des compléments ou des propositions entières, il rapproche ici les deux verbes, car il renonce à l'inversion simple, normale dans les propositions locatives, rejetant ainsi le premier verbe à la fin de la proposition. Grâce à ce procédé, les deux verbes se trouvent immédiatement avant et après la conjonction et, ce qui met en relief leur lien intime.

La même combinaison du passé composé et du présent se trouve dans une coordination dont la première proposition est négative et la seconde contient 
la locution de restriction ne...que.

Je n'ai jàmais aimé la demeure ... et ne songe qu'a passer outre dès que ternit la nouveauté. (1429)

La proposition à valeur restrictive complète le sens de l'action négative indiquée dans la premiere proposition; naturellement, l'ellipse du sujet renforce ce lien. Le rapport temporel: passé composé - présent indique que $I^{\prime}$ attitude du personnage demeure dans le présent telle qu'elle était dans le passé.

La distance temporelle est encore plus accentuée dans la phrase suivante où nous rencontrons le plus-que-parfait et le présent et où une proposition négative est suivie d'une proposition affirmative. Ariane affirme que Thésée lui a fait des promesses, mais lui commente:

Je n'avais rien promis du tout et tiens surtout à rester libre. (1429)

Malgré la distance temporelle, il y a un lien entre les deux actions qui est accentué par l'ellipse du second sujet: l'action du verbe au plus-queparfait est motivée par le verbe au présent qui exprime une attitude constante de Thésée - s'il n'avait rien promis, c'est qu'il tenait, qu'il tient depuis toujours à rester libre.

Voilà une autre phrase où le premier verbe est à l'imparfait, voix passive, le second au présent:

$J$ 'étais surpris par tous les sens et ne puis dire combien les Crétois me paraissaient étrangers. (1422)

Le rapport causal entre les deux verbes est bien visible, celui au présent étant la conséquence de celui à l'imparfait ${ }^{8}$.

8 Cf. Ferdinand Brunot: "Le rapport de cause est, dans le langage, intimement lié au rapport de suite dans le temps. Un fait qui s'est développé après un autre apparaît comme le résultat de cet autre" (op. cit., p. 812). 
Plus problématique est une phrase où le premier verbe est au futur, le second, après l'ellipse du sujet, au présent:

Je saurai faire respecter les lois; me faire respecter, sinon craindre, et prétends que l'on puisse dire ... (1446)

On peut justifier l'emploi de ces deux temps par le fait que le verbe prétendre exprime un acte de volonté, qu'il est donc orienté vers le futur. La distance temporelle entre les deux verbes est adoucie par trois propositions infinitives qui dépendent du premier verbe et le séparent du second.

Deux temps différents sont employés aussi dans une coordination adversative:

Quant à moi je le suivais à peine, déconcerté par trop de nouveauté; mais ne laissai pas d'être surpris par la souplesse ... des acrobates ... (1423)

Le verbe à l'imparfait exprime une action prolongée (je le suivais à peine), qui est partiellement niée dans la seconde proposition (mais ne laissai d'être surpris) dont le verbe au passé simple indique une réaction qui n'est que de brève durée. Il faut noter le parallélisme des compléments qui suivent chaque verbe: déconcerté par trop de nouveauté - surpris par la souplesse des acrobates.

Deux modes différents sont employés dans une coordination adversative où la conjonction employée est toutefois:

... on devrait savoir ... que Minos ne tient à l'ordinaire aucun compte des rangs sociaux, grades ou hiérarchies; toutefois se sentirait, certes, flatté lorsqu' un prince éminent comme toi voudrait bien s'intéresser à son fils. (1441)

Il s'agit de deux propositions substantives mais coordonnées et qui sont en forte opposition. Cette opposition est mise en valeur par plusieurs procéclés: emploi de la conjonction toutefois, plus rare et plus expressive 
que la conjonction mais; contraste dans la forme des deux propositions: la seconde affirme ce que la première nie; omission du pronom sujet qui rapproche les deux verbes; emploi de deux modes différents dont le second, le conditionnel, admet le doute sur la réalité de l'action qui est opposée à celle exprimée par le premier verbe, mais ce doute est aussitôt effacé par l'adverbe certes; emploi du point-virgule qui marque l'opposition d'une manière plus nette que la simple virgule.

Ordinairement l'ellipse du pronom sujet produit ses effets au sein d'une phrase. Mais il arrive aussi que ces effets se répetènt d'une phrase à l'autre, à travers un passage entier. Prenons comme exemple le passage où Pasiphaë essaie de séduire Thésée et de le faire désister de son projet de tuer Minotaure. Dans cette double intention elle prononce, séparées par un discours narratif de Thésée, deux phrases, l'une se rapportant à elle-même, l'autre à Minotaure. Leur construction est strictement parallèle: deux fois nous avons la coordination copulative avec et; deux fois les deux propositions coordonnées sont négatives, deux fois la seconde proposition renforce le sens de la première:

Je ne suis pas ce que vous croyez et n'en veux nullement à votre personne ... (1426)

Je ne sais ce qu'on a pu vous raconter de lui et ne tiens pas à le savoir. (1427)

Voilà encore l'alinéa où Thésée raconte combien il a peiné avant de faire sortir ses compagnons du labyrinthe. L'ellipse du sujet dans les coordinations y apparaît non moins de sept fois:

Lorsque je fis mine de les emmener, ils protestèrent qu'ils étaient fort bien là et ne songeaient nullement à partir. $J$ 'insistai, dis que je leur apportais la délivrance. "La délivrance de quoi ?" s'écrièrent-ils; et, ligués soudain 
contre moi, m'injurièrent. Je m'attristais grandement, à cause de Pirithoüs. Il me reconnaissait à peine, reniait la vertu, se rigolait de sa propre valeur et proclamait sans ve rgogne qu'il ne consentirait à quitter le bien-êtrè présent pour toute la gloire du monde. Je ne pouvais cependant lui en vouloir, sachant trop que, sans la précaution de Dédale, j'aurais sombré de même, fait "chorus avec lui, avec eux. (1440)

Nous y rencontrons d'abord trois coordinations dont chacune comprend deux propositions. Dans la prèmiere de ces coordinations deux propositions subordonnées substantives sont coordonnées: qu'ils étaient fort bien là et ne songeaient nullement à partir. La seconde proposition qui est négative indique une conséquence de la première. Dans la deuxième coordination (J'insistai, dis ...), la deuxième action suit immédiatement la première ou plutôt s'insère en elle. Dans la troisième coordination (s'écrièrent-ils; et ... $\mathrm{m}$ 'injurièrent) il $\mathrm{y}$ a $\mathrm{l}^{\prime}$ 'inversion du sujet dans la première proposition; une telle différence dans la construction des deux propositions exige normalement la répétition du sujet. Omettant la répétition, Gide souligne la parenté entre les deux actions. L'effet de cet écart grammatical est adouci par l'insertion d'un long complément participial entre les deux verbes (ligués soudain contre moi). Suit la description du comportement de Pirithoüs, quatre propositions coordonnées exprimant quatre actions qui se suivent immédiatement ou s'entremêlent: Il me reconnaissait à peine, reniait la vertu, se rigolait de sa propre valeur et proclamait sans vergogne ... Le sujet commun n'est exprimé que dans la première proposition. L'identité logique de deux actions est exprimée dans la dernière phrase où Thésée fait des suppositions sur son propre comportement probable et où il y a la non-répétition du pronom sujet et de l'auxiliaire dans le conditionnel passé: ... j'aurais sombré de même, fait chorus avec lui, avec eux. 
Le très grand nombre de coordinations où le pronom sujet n'est pas répété montre la, tendance de l'auteur de Thésée de mettre l'accent sur le verbe, sur l'action. Il en résulte un style dynamique, mais aussi un style serré, car l'ellipse du pronom sujet met en relief d'une manière synthétique les liens logiques ou affectifs entre les verbes, insistant souvent sur des nuances très subtiles de l'action. Cette union entre les verbes est produite par deux procédés tres différents, voire opposés: 1'abolition, au moyen de l'ellipse du sujet, de la distance entre les verbes, proches déjà par leur position dans la phrase - l'établissement de la distance entre les verbes au moyen des compléments ou des phrases intercalés, I'ellipse du sujet produisant ainsi un arrêt, une pause de réfléxion qui invite le lecteur a revenix au premier verbe et à chercher son lien avec les suivants. Les effets stylistiques sont encore plus forts lorsque le pronom sujet est omis dans des situations grammaticales où le bon usage du français moderne demande sa répétition, notamment avec des verbes à des temps ou modes différents ou quand il y a l'inversion du sujet. Ia liberté que Gide se permet dans de tels cas a ses racines dans sa bonne connaissance du français classique, dans son admiration des auteurs classiques. Le pronom sujet ètait très souvent absent en ancien français où les terminaisons verbales, étant sonores, indiquaient suffisamment les personnes grammaticales ${ }^{9}$. Il était encore fréquemment omis au $\mathrm{XV}^{\mathrm{e}}$ et $\mathrm{XVI}^{\mathrm{e}}$ siècles. Le $\mathrm{XVII}^{\mathrm{e}}$ siècle conservait la facilité de ne pas répéter le sujet dans certains cas où aujourd'hui la répétition est normale, p. ex. quand il s'agit des temps différents dans la coordination ${ }^{10}$. Ferdinand

9. Cf. Maurice Grevisse, op. cit., pp. 164 et 402 , et Lucien Foulet, Petite syntaxe de l'ancien francais, Paris, Honoré Champion, 1930, pp. 196-198. Foulet montre que $1^{\prime}$ absence du pronom sujet dans les propositions principales est très fréquente en ancien français parce que 1 'inversion du sujet qui entraîne facilement son omission est "le grand fait qui domine la construction médiévale" (op. cit., p. 307, pp. 313-314).

10 Maurice Grevisse, op. cit., p. 164, allègue l'exemple suivant: $\mathrm{J}^{\prime}$ ignore tout le reste, Et venais vous conter ce désordre funeste (Racine, Athalie, II, 2). 
Brunot dit à propos des auteurs modernes qui omettent la répétition du sujet dans de tels cas: "Ces cas, assez rares d'ailleurs, ne sont que des archaïsmes ou des fantaisies" ${ }^{11}$. On peut parler, à propos de Thésée, des archaïsmes, sans doute, peut être aussi des fantaisies, mais fantaisies heureuses qui lui confèrent un style mouvementé, aisé et riche en nuances; ce style qui a permis au Gide vieillissant de faire jaillir dans son dernier récit son intarissable humour, son immense amour de la vie et aussi sa résignation aux approches de la mort.

\section{Povzetek}

\section{STILISTIČNA UČINKOVITOST IZPUSTA PRONOMINALNEGA SUBJEKTA V GIDOVI PRIPOVEDI "TEZEJ"}

V svoji zadnji pripovedi Tezej (1944) uporablja André Gide poleg drugih stilističnih sredstev tudi izpust pronominalnega subjekta $\mathrm{v}$ priredjih, kjer je ta skupen več glagolom. Subjekt je tedaj izražen $v$ obliki samostalnika ali osebnega zaimka $\mathrm{v}$ prvem stavku priredja, $\mathrm{v}$ ostalih pa se ne ponavlja kot osebni zaimek. Gide pogosto izpušča subjekt $v$ skladu z normirano rabo moderne francoščine, včasih pa tudi na zelo samosvoj način, zlasti kadar so glagoli priredja $v$ različnih časih in naklonih, ali kadar sta povezana nikalni in trdilni stavek, ali kadar je $\mathrm{v}$ prvem stavku besedni red osebka in glagola obrnjen. Pogosten in celo nenavaden izpust subjekta $v$ priredjih daje poseben poudarek glagolom in njihovi medsebojni povezavi. Zato se Gidov slog v pripovedi Tezej odlikuje po razgibanosti, zgoščenosti in bogastvu logičnih in čustvenih odtenkov.

11 Ferdinand Brunot, op. cit., p. 280. Brunot y cite une phrase de Flaubert: Son père ... écrivit, en fournissant les explications les plus précises, et terminait sa lettre par une badinerie. 